\title{
BMJ Open A mixed methods feasibility study to evaluate the use of a low-intensity, nurse-delivered cognitive behavioural therapy for the treatment of irritable bowel syndrome
}

\author{
Andrew David Dainty, ${ }^{1}$ Mark Fox ${ }^{2}$ Nina Lewis, ${ }^{3}$ Melissa Hunt, ${ }^{4}$ Elizabeth Holtham, ${ }^{5}$ \\ Stephen Timmons, ${ }^{6}$ Philip Kinsella, ${ }^{7}$ Andrew Wragg, ${ }^{8}$ Patrick Callaghan ${ }^{6}$
}

To cite: Dainty $A D$, Fox $M$, Lewis N, et al. A mixed methods feasibility study to evaluate the use of a lowintensity, nurse-delivered cognitive behavioural therapy for the treatment of irritable bowel syndrome. BMJ Open 2014;4:e005262.

doi:10.1136/bmjopen-2014005262

- Prepublication history for this paper is available online. To view these files please visit the journal online (http://dx.doi.org/10.1136/ bmjopen-2014-005262)

Received 14 March 2014 Revised 15 May 2014 Accepted 2 June 2014

\section{CrossMark}

For numbered affiliations see end of article.

Correspondence to Andrew David Dainty; ntxad9@nottingham.ac.uk

\section{ABSTRACT}

Introduction: Irritable bowel syndrome (IBS) is characterised by symptoms such as abdominal pain, constipation, diarrhoea and bloating. These symptoms impact on health-related quality of life, result in excess service utilisation and are a significant burden to healthcare systems. Certain mechanisms which underpin IBS can be explained by a biopsychosocial model which is amenable to psychological treatment using techniques such as cognitive behavioural therapy (CBT). While current evidence supports CBT interventions for this group of patients, access to these treatments within the UK healthcare system remains problematic.

Methods and analysis: A mixed methods feasibility randomised controlled trial will be used to assess the feasibility of a low-intensity, nurse-delivered guided self-help intervention within secondary care gastrointestinal clinics. A total of 60 participants will be allocated across four treatment conditions consisting of: high-intensity CBT delivered by a fully qualified cognitive behavioural therapist, low-intensity guided self-help delivered by a registered nurse, self-help only without therapist support and a treatment as usual control condition. Participants from each of the intervention arms of the study will be interviewed in order to identify potential barriers and facilitators to the implementation of CBT interventions within clinical practice settings. Quantitative data will be analysed using descriptive statistics only. Qualitative data will be analysed using a group thematic analysis.

Ethics and dissemination: This study will provide essential information regarding the feasibility of nursedelivered CBT interventions within secondary care gastrointestinal clinics. The data gathered during this study would also provide useful information when planning a substantive trial and will assist funding bodies when considering investment in substantive trial funding. A favourable opinion for this research was granted by the Nottingham 2 Research Ethics Committee.

Trial registration number: ISRCTN: 83683687 (http://www.controlled-trials.com/ISRCTN83683687).

\section{Strengths and limitations of this study}

This study will provide essential feasibility data regarding the use of nurse-delivered, lowintensity cognitive behavioural therapy (CBT) treatment approaches with secondary care gastrointestinal clinics.

- The mixed methods study design will produce qualitative and quantitative data for analysis which will provide a range of feasibility data.

- The study will not collect economic or service use data, although this should ideally be a concern of a follow-on study.

- This study does not consider the application of other CBT treatment mechanisms such as groupbased or internet therapy.

\section{BACKGROUND}

Irritable bowel syndrome (IBS) is a functional gastrointestinal (GI) disease that is characterised by the presence of altered bowel habit (diarrhoea and constipation) and the presence of symptoms including bloating and abdominal pain. ${ }^{1}$ The cause of IBS remains unexplained, although it is known that a proportion of patients will develop IBS following an initial insult such as GI infection. ${ }^{2}{ }^{3}$ Research has also established that food intolerance, the balance of gut microflora and certain changes within the immune system might be underlying mechanisms in IBS. $^{4} 5$ However, routine investigations fail to identify abnormal pathology for IBS and patients are left with a range of symptoms which are managed empirically by antispasmodics, low-dose antidepressants and other medications. The daily activities of patients with IBS are often adversely affected and patients may be troubled by concerns related to the cause 


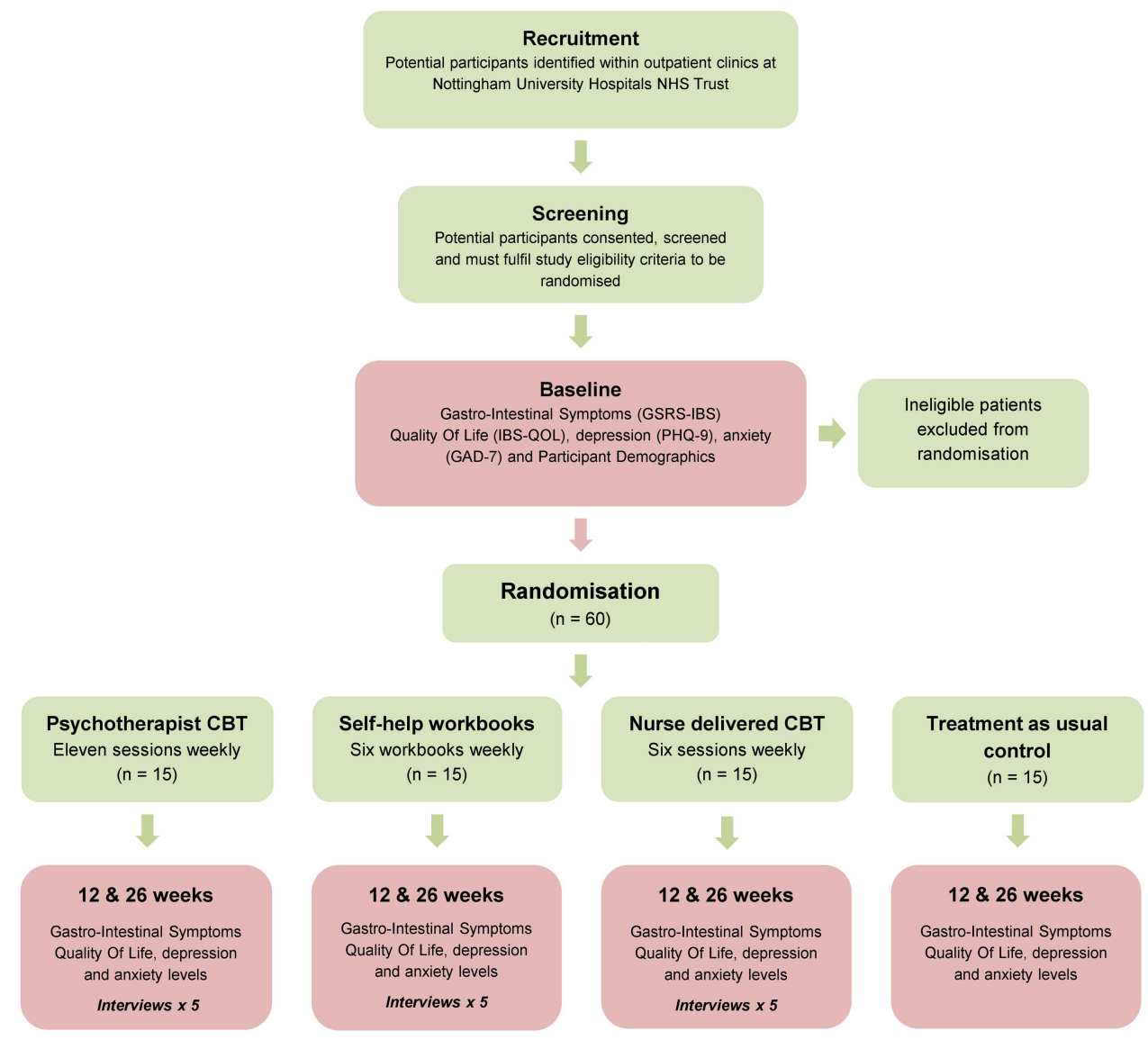

Figure 1 Study flow diagram detailing numbers of participants and participant flow through the study-related procedures and follow-up data collection periods. CBT, cognitive behavioural therapy; GSRS-IBS, Gastrointestinal Symptom Rating Scale for irritable bowel syndrome.

and the effects of their condition. ${ }^{6}$ Patients with IBS tend to report a poor quality of life (QOL) and often experience absenteeism. ${ }^{7}$ Moreover, IBS places a significant strain on healthcare resources and service utilisation, with patients with IBS consuming over $50 \%$ more resources than carefully matched controls. ${ }^{89}$ Health professionals may also find themselves challenged with the management of such a complex and persistent disorder. $^{10}$

\section{Psychological aspects of IBS}

Psychosocial factors and stress are related to the maintenance and manifestation of IBS symptoms in many patients. ${ }^{11}$ Indeed, historically, clinicians felt that IBS was purely a physical manifestation of emotional problems. ${ }^{12}$ In recent years, the association IBS has with psychological issues has been supported with experimental data. For example, Guthrie et al $\mathrm{s}^{13}$ study involving 107 participants demonstrates that the perception of bowel sensations may relate directly to psychological distress. The literature reports a prevalence of psychological comorbidity ranging anywhere between 50\% and 90\% for common mental health problems such as anxiety and depression. ${ }^{14}$ Traumatic events or stressful experiences which shape an individual's life experiences may also play a fundamental role in IBS. Indeed, it is not uncommon for patients with IBS to report some history of physical or sexual abuse. ${ }^{15}{ }^{16}$ Stressful life events, in general, are associated with symptom onset and severity. ${ }^{17}$ These psychological factors and the complex manifestation of IBS symptoms are widely acknowledged as a disease process which involves an individual's social environment, physiology and psychology. ${ }^{11}$ These elements form part of the 'biopsychosocial model' which offers a holistic and rational explanation for the presentation and persistence of IBS symptoms in many individuals.

Because the mechanisms of IBS can be explained by a biopsychosocial model, effective treatment approaches may include psychological interventions such as cognitive behavioural therapy (CBT). Best practice guidelines suggest that CBT, hypnotherapy and psychotherapy are useful interventions for patients with IBS. ${ }^{18}$ CBT is particularly useful for addressing negative thought patterns ${ }^{6}$ and catastrophising cognitions and avoidant behaviours. ${ }^{19}$ CBT can also be used to target maladaptive thought processes ${ }^{20}$ and help patients understand the interactions between their thoughts and their symptoms. ${ }^{21}$ CBT is well supported empirically as an effective intervention for patients with IBS. ${ }^{22-24}$ Although the 
results of many of these investigations are promising, a recent systematic review identified issues regarding the methodological quality and power of trials which support these interventions. ${ }^{25}$

\section{Rationale}

There is a need for CBT to be further evaluated using robust methods. ${ }^{14}$ Furthermore, there is a need for future research to evaluate psychological therapies which address concerns regarding treatment provision and accessibility. ${ }^{26}$ Many patients within the National Health Service (NHS) healthcare system have no routine access to psychological interventions for the treatment of their IBS symptoms. Notwithstanding these concerns, there is encouraging activity within the wider UK healthcare system to suggest that the government and policymakers are beginning to recognise the impact that psychological therapies such as CBT have on improving health and reducing loss of productivity. ${ }^{27}$ This study will evaluate the feasibility and acceptability of a novel, nurse-delivered, low-intensity CBT, guided self-help intervention for the treatment of IBS. It is hoped that the nursing intervention will provide a feasible mechanism for the delivery of CBT which may be economically viable, more accessible to patients and implementable within the UK healthcare system. Qualitative research performed during the course of this study will provide researchers and funding bodies with essential information on the acceptability and practical application of CBT interventions in patients with IBS.

\section{METHODS AND ANALYSIS}

\section{Study aim}

To carry out a feasibility study to explore the use of various CBT treatment delivery mechanisms for patients with IBS within secondary care GI clinics.

\section{Study objectives}

- To develop a tailored, nurse-delivered CBT treatment for patients with IBS.

- To examine the number of patients needed to screen in relation to the numbers successfully randomised into the study.

- To gather reasons for patients not wishing to take part in CBT treatment approaches for their IBS.

- To explore the experience of participants undergoing the study's treatment conditions.

- To identify barriers and facilitators to the implementation of the trial interventions within real clinical settings.

- To measure the follow-up and response rates to study questionnaires.

- To describe the range of data with relevant descriptive statistics.

- To test the data collection procedures and time required to collect study data.

\section{Study design}

The randomised controlled trial (RCT) is considered one of the most powerful tools used in research to establish new knowledge claims. ${ }^{28} 29$ The proposed RCT will establish the cause and effect relationship between CBT and patient outcomes. A mixed methods research (MMR) approach, underpinned by a pragmatic philosophy, will draw from the strengths of qualitative and quantitative approaches. ${ }^{30}$ MMR is defined as "collecting, analysing and mixing both quantitative and qualitative data in a single study or series of studies." ${ }^{\text {31 }}$ The growing popularity of MMR approaches would suggest that many researchers now recognise the value of this pragmatic approach. ${ }^{32}$ MMR provides robust and rigorous approaches to research ${ }^{33}$ and creates a richer understanding of phenomena. ${ }^{34}$ Many of the RCTs which have investigated the use of CBT for patients with IBS lack a qualitative research component. This limits our understanding of the suitability and acceptability of these interventions for this group of patients. The incorporation of qualitative methods will ensure the research considers problematic moments and meanings in individuals lives. ${ }^{35}$ These methods will also contextualise the findings of the research ${ }^{36}$ and enable trial participants to provide information regarding their responses to quantitative variables. ${ }^{37}$

\section{Patients}

Around $60 \%$ of patients referred to GI clinics with lower GI symptoms are diagnosed with IBS. ${ }^{38}$ Adults (over 18 years of age) have been chosen as the main group of patients for inclusion as IBS is typically associated with the third and fourth decades of life. ${ }^{39}$ In order to ensure that participants have symptoms consistent with IBS, they will be screened and required to fulfil Rome III criteria. This tool has been developed by the Rome Foundation with a long-standing history of development and a strong evidence base. ${ }^{40}$ It has been successfully utilised as symptomatic criteria for IBS in a number of RCTs $^{22} 4142$ and is being constantly updated and reviewed in the light of changes in the recent literature. ${ }^{43}$ Participants with symptoms inconsistent with IBS according to Rome III criteria will be excluded from randomisation.

\section{Inclusion criteria}

- Adult male and female patients aged 18 years or older at the time of enrolment.

- Documented medical diagnosis of IBS.

- Patients with IBS which meets Rome III criteria.

- Able to read, write and speak English.

- Able to provide written informed consent.

- Patients with and without concomitant antidepressant use.

- Able to commit to weekly treatment sessions within the intervention arms of the study. 


\section{Exclusion criteria}

- Already receiving psychological therapy or hypnotherapy.

- Existing diagnosis of bowel disease based on endoscopic or histological criteria (ie, Crohn's disease, ulcerative colitis, coeliac disease).

- Presence or history of structural or surgical diseases of the GI tract (not including appendix or gall bladder surgery).

- Evidence of alcohol or substance misuse.

- An established cause for bowel symptoms other than IBS (ie, medication use).

- The presence of suicidal ideation or self-harm.

- Significant psychiatric comorbidity (schizophrenia, bipolar disorder, obsessive compulsive disorder).

- Currently taking part in other research studies.

\section{Intervention}

Many studies which have investigated the use of CBT for patients with IBS utilise high-intensity treatment protocols. Several RCTs have used 10-week treatment programmes, ${ }^{22} 264445$ with most sessions over $50 \mathrm{~min}$ in duration. Some researchers have experimented with much lower intensity treatment methods and have altered delivery mechanisms in order to improve the provision of CBT interventions such as internet CBT. ${ }^{19}$ However, there is concern regarding the delivery of online interventions relating to confidentiality, a loss of visual and auditory clues during therapy and a lack of suitability for managing crisis situations. ${ }^{46}$ A recent survey of 658 patients suggests that internet-delivered CBT would be unacceptable to over $40 \%$ of those surveyed. ${ }^{47}$ Other delivery mechanisms include group-based treatments, ${ }^{42}$ although some authors suggest that individual contact is better suited to tailoring therapy to the needs of the client. ${ }^{48}$ CBT has been effective when delivered by trained nurses within primary care settings. ${ }^{24}$ It is therefore possible that similar effects might be achievable if therapy were to be delivered by GI nurses within secondary care utilising a lower intensity, guided self-help treatment approach which could be less time consuming to deliver and further increase access to evidencebased interventions for patients.

Registered nurses working within gastroenterology are well placed to deliver psychotherapeutic interventions to these groups of patients as they are familiar with the complexity of chronic GI symptoms and the impact they have on patients and their daily activities. The preparatory training utilised within this study offers a replicable mechanism for expansion of the professional scope of nursing practice for nurses working with the GI specialty, while increasing access to evidence-based intervention patients may not otherwise receive.

We have adapted the protocol and self-help materials originally developed by Hunt $e t a l^{19}$ at the University of Pennsylvania USA, while working in collaboration with service users to maximise the quality and suitability of our nursing intervention. The nursing intervention consists of an initial $60 \mathrm{~min}$ assessment session, with five further $30 \mathrm{~min}$ treatment sessions over a 6 -week treatment period. Participants will work through six guided self-help treatment modules with a nurse therapist, consisting of:

- an introduction to IBS,

- relaxation training,

- the cognitive model of stress management,

- the cognitive model of IBS and behavioural experiments,

- managing avoidance using exposure,

- diet and IBS.

The nurse therapist (ADD) undertook the first two modules of the National Curriculum for the education of Psychological Wellbeing Practitioners ${ }^{49}$ in order to prepare for the nurse therapist's role. This training consisted of 25 days of university-based teaching sessions and simulated clinical skills training. The therapist completed all assessments for these modules to the nationally accredited assessed standard, but did not access the supervised practice hours within a mental health services placement as this was not felt to be necessary or economically viable for the preparation of the nurse therapist's role for the treatment of IBS.

\section{Comparators}

The nursing intervention will be compared to: (A) the services of a fully trained and experienced cognitive behavioural therapist using high-intensity treatment; (B) a six module, CBT-based self-help workbook as a stand-alone intervention; and (C) a treatment as usual (TAU) control condition. Participants in all four treatment conditions will be permitted to continue with the routine medical management of their IBS.

\section{Psychotherapist}

Comparing the nursing intervention with the services of a cognitive behavioural therapist during a substantive trial will help establish whether the study's nursing intervention is as effective and acceptable as current evidence-based treatment approaches. The psychotherapist will deliver treatment to participants using a protocol developed from the work of Toner et al, ${ }^{48}$ not dissimilar to previous trials of $\mathrm{CBT}^{23}$ Participants will attend 11 treatment sessions at weekly intervals, the first $2 \mathrm{~h}$ in duration and subsequent sessions will last for up to an hour.

\section{Self-help workbooks}

Participants will be given the same six module self-help treatment workbooks as used within the nursing intervention. However, in order to identify any additional benefit that the nurse therapist has within the study's nursing intervention, the treatment workbooks will be used as a stand-alone treatment. Participants will be given the workbooks by the nurse therapist and advised that they should work through the modules on a weekly basis. No further therapist support will be provided. 


\section{Treatment as usual}

A TAU condition will enable the study of the intervention in comparison to current treatment approaches and may also be required for economic evaluations within a definitive study. It is hoped that the feasibility study will provide further information regarding the suitability of a TAU control condition.

\section{Outcomes}

The literature would suggest that an extended period of follow-up is necessary in order for researchers to establish whether the positive treatment effects of CBT diminish over time. ${ }^{25}$ However, longer follow-up periods are not feasible within the scope of this $\mathrm{PhD}$ project and will be the concern of a definitive trial. Similarly, although an important feature of a definitive study may be a costeffectiveness data collection and analysis, a limitation is that such data will not be collected during this feasibility work. All outcome measures will be assessed at baseline, and at 12 and 26 weeks postrandomisation. The study flow diagram (figure 1) demonstrates the various data collection points throughout the study period.

\section{IBS symptom severity}

Numerous studies have identified the negative impact that IBS symptoms have on QOL, ${ }^{50} 51$ and suggest that symptoms are largely responsible for patients seeking consultation. ${ }^{42}$ IBS symptom severity will be the primary outcome for this study and will be measured with participant responses on the Gastrointestinal Symptom Rating Scale for IBS (GSRS-IBS). ${ }^{52}$ GSRS-IBS consists of 13 items with five symptom subdomains including: abdominal pain, bloating, diarrhoea, constipation and satiety. Participants are asked to record the previous 7 days' symptoms indicating responses on a seven-point Likert scale. Mean item scores (between 1 and 7) are calculated for each of the five subdomains. Treatment responders will be defined by a reduction in IBS symptom scores of more than $50 \%$.

\section{Quality of life}

IBS has also been shown to impact on QOL to a similar degree as chronic conditions such as diabetes mellitus. ${ }^{51}$ Researchers have suggested that the measurement of QOL within therapeutic trials for IBS is clearly warranted.$^{50}$ QOL will therefore be included as a secondary outcome measure utilising IBS-QOL. ${ }^{53}$ IBS-QOL consists of 34 self-report Likert scales specific to IBS. IBS-QOL also makes possible the analysis of eight subdomains which include: dysphoria, interference with activity, body image, health worry, food avoidance, social reaction and sexual relationships.

\section{Comorbid anxiety and depression}

Much of the impact that IBS has on QOL is explained by psychological factors ${ }^{7}$ which, when treated, may reduce IBS-related symptoms. ${ }^{54}$ Levels of depression will be measured with the Patient Health Questionnaire
(PHQ) $-9,{ }^{55}$ and levels of anxiety using the Generalised Anxiety Disorder (GAD) $-7 .{ }^{56}$ Both of these well-validated measures consist of nine-item and seven-item self-report Likert scales, respectively, indicating depression and anxiety severity scores. Necessary permissions have been sought for all measures utilised during the conduct of this research.

\section{Randomisation and blinding}

Participants will be allocated to one of four treatment conditions using random permuted blocks. The principal investigator will screen all potential participants and randomise eligible participants using an online randomisation system (see http://www.sealedenvelope.com). All quantitative, postintervention outcome data will be collected by a research assistant blind to the allocation of participants. The study's investigators will be aware of the allocation of study participants.

\section{Sample size}

This study is concerned with generating descriptive statistics that will be used to evaluate the feasibility of the proposed methods and not to establish the effectiveness or generalisability of the interventions. A pragmatic decision was made to randomise 60 participants across four treatment conditions. Approximately 15 participants will be assigned to each of the study conditions (see study diagram).

\section{Recruitment and participant selection}

Potentially eligible clinic patients will be identified by a member of their care team and given participant information packs or will be sent information relating to the trial via their hospital physician. Participants may express an interest in taking part in the research study by returning a reply slip using a prepaid postage service. Posters will also be deployed on clinic and hospital notice boards. We will also write to potentially eligible participants on our Biomedical Research Unit research database.

\section{Data collection}

The quantitative measures will be collected by the principal investigator (ADD) at screening and sent to participants by mail at 12 and 26 weeks postrandomisation. Postintervention outcome data will be returned to a research assistant blind to the allocation of participants. Interview data will be collected by a coinvestigator (EH) not connected to the delivery of the trial interventions.

The qualitative element of the study aims to capture the knowledge which is located in the minds and personal experiences of others. ${ }^{57}$ Although some authors advocate unstructured interview techniques, ${ }^{58}$ a semistructured approach will ensure that information relating to the phenomena of interest is obtained. ${ }^{59}$ Furthermore, the prestructure has provided a mechanism for the involvement of our patient advisory group, which has helped shape the contents of the interview schedule. The structure and content of the interviews 
was initially developed as a result of the researcher's prior knowledge of GI research and the subject area as a result of a literature review. Interview questions will be used by the interviewer to seek clarification, illustration and further exploration regarding important issues. ${ }^{60}$ The qualitative element of our study aims to evaluate the experiences of service users undergoing the three trial treatment protocols in order to identify potential barriers and facilitators to the use of the interventions. Five participants from each of the intervention arms of the study will be recruited to qualitative interviews. This study has also received ethical permission to make enquiries into the reasons patients may have for not taking part in the research. It will be made clear that patients are under no obligation to provide this information to the research team. This information will be useful for helping researchers understand barriers to the successful implementation of psychological therapies for IBS within the NHS.

\section{Data analysis}

\section{Quantitative data}

Quantitative data will be analysed descriptively. Measures of mean and variance including CIs and SD will be used to describe the full range of data at baseline and at follow-up. An intention to treat analysis will be facilitated as missing data will be rectified using the last observation carried forward. Outcome-related data will be analysed using SPSS version 22.

\section{Qualitative data}

Corroboration will be sought between qualitative and quantitative elements of the study. ${ }^{61}$ Qualitative data will be generated in the form of interview transcripts for analysis. The qualitative data will supplement the quantitative outcome data by identifying convergence and differences between the two databases. ${ }^{62}$ Interview recordings will be transcribed verbatim prior to conducting the framework analysis advocated by Ritchie and Spencer. ${ }^{63}$ In order to improve the rigour during the analysis and guard against investigator bias, a group analysis approach will be used to analyse the interview transcripts. ${ }^{64}$ The study's principal investigator and the interviewer will jointly conduct the thematic analysis of the interview transcripts supervised by an experienced qualitative researcher.

\section{Patient and public involvement}

Patient and public involvement (PPI) is defined by the National Institute for Health Research (NIHR) as "research that is done with or by the public and not to, about or for them." ${ }^{65}$ Many awarding bodies expect to see evidence of PPI within major grant applications. ${ }^{66}$ Our research team has developed an excellent working alliance with a group of six patient/public volunteers who have helped shape and improve the design of the study. The patient advisory group has also helped to review study documents and highlight potential barriers to the recruitment and selection of participants. By involving service users in the conduct of the research, it is hoped that equilibrium has been sought in order to address any power imbalances between health professionals and patients. ${ }^{67}$ It is also hoped that PPI has helped maximise the quality of our nursing intervention.

\section{Ethical issues}

Psychological issues and IBS

Although not all participants recruited for this study will have a psychological comorbidity, some participants may not accept, or may even reject, a psychological element to their IBS. ${ }^{68}$ It is hoped that by conducting interviews with study participants, barriers to the implementation of psychological therapies relating to these phenomena will be further explored.

\section{Implications for participants taking part in a trial of psychological interventions}

Some participants may be concerned regarding the implications of receiving psychotherapy during the trial. The emphasis within our study is to focus on helping patients to live with the impact of their IBS symptoms rather than specifically targeting those only with psychological comorbidity (such as underlying anxiety or depression). It will be made clear when writing to the participant's doctor that participation in our study does not necessarily suggest or confirm that participants are psychologically unwell. This may be particularly important where a diagnosis of psychological illness may have negative implications for the participant, that is, those with rigorous occupational requirements. However, levels of psychological illness will be recorded during a participant's journey through the study as we are interested in the effects that treatment may have on these underlying issues. We will ensure that participants are made fully aware of these issues during recruitment and selection and that these details are included within the study information sheets. A participant's hospital care team and general practitioner will be notified where a moderate-to-severe level of anxiety or depression is detected during measurement, as per current practice within our GI clinics.

Host organisation approval was sought from the Nottingham University Hospitals NHS Trust prior to the start of any research-related activity.

\section{Author affiliations}

${ }^{1}$ Nottingham Digestive Disease Centre Biomedical Research Unit and School of Health Sciences, University of Nottingham, Nottingham, UK

${ }^{2}$ Functional GI Diagnostics Laboratory, Division of Gastroenterology and Hepatology, Centre for Reflux and Swallowing Disorders, University Hospital, Zürich, Switzerland

${ }^{3}$ Nottingham University Hospitals NHS Trust, Nottingham, UK

${ }^{4}$ University of Pennsylvania, Philadelphia, PA, USA

${ }^{5}$ Faculty of Medicine \& Health Sciences, University of Nottingham, Nottingham, UK

${ }^{6}$ School of Health Sciences, University of Nottingham, Nottingham, UK 
${ }^{7}$ Institute of Mental Health, University of Nottingham, Nottingham, UK ${ }^{8}$ Nottingham Digestive Disease Centre Biomedical Research Unit, Nottingham University Hospitals, Nottingham, UK

Acknowledgements An adapted version of the irritable bowel syndrome self-help intervention originally developed by Dr Melissa Hunt at the University of Pennsylvania is being used within this study, as permitted under a research license issued by the University of Pennsylvania, Philadelphia, USA.

Contributors ADD developed the initial protocol draft. PC, MF, PK and NL were responsible for the development, refinement and approval of the study protocol. PC and PK provided mental health expertise while physicians MF and NL provided gastrointestinal expertise for the protocol development. ADD is conducting the project as part of his PhD studies. MH and ADD collaborated on the adaptation and development of the nurse-delivered treatment protocol and associated materials. ADD and EH developed the qualitative elements of the protocol which is supervised and edited by ST. AW is the study's patient and public involvement manager responsible for facilitating patient and public involvement in the development of the study. All authors have approved the final version of this manuscript.

Competing interests ADD's PhD studentship was funded by the University of Nottingham and the NIHR Gastrointestinal Biomedical Research Unit at Nottingham University Hospitals NHS Trust.

Ethics approval Nottingham 2 Local Research Ethics Committee.

Provenance and peer review Not commissioned; externally peer reviewed.

Open Access This is an Open Access article distributed in accordance with the Creative Commons Attribution Non Commercial (CC BY-NC 4.0) license, which permits others to distribute, remix, adapt, build upon this work noncommercially, and license their derivative works on different terms, provided the original work is properly cited and the use is non-commercial. See: http:// creativecommons.org/licenses/by-nc/4.0/

\section{REFERENCES}

1. Schmulson M, Chang L. Diagnostic approach to the patient with irritable bowel syndrome. Am J Med 1999;107:20-6.

2. Spiller R, Garsed K. Postinfectious irritable bowel syndrome. Gastroenterology 2009;136:1979-88.

3. DuPont A. Postinfectious irritable bowel syndrome. Clin Infect Dis 2008;46:594-9.

4. Yang J, Fox M, Cong $\mathrm{Y}$, et al. Lactose intolerance in irritable bowel syndrome patients with diarrhoea: the roles of anxiety, activation of the innate mucosal immune system and visceral sensitivity. Aliment Pharmacol Ther 2014;39:302-11.

5. O'Mahony L, McCarthy J, Kelly P, et al. Lactobacillus and bifidobacterium in irritable bowel syndrome: symptom responses and relationship to cytokine profiles. Gastroenterology 2005;128:541-51.

6. North C, Hong B, Alpers D. Relationship of functional gastrointestinal disorders and psychiatric disorders: implications for treatment. World J Gastroenterol 2007;13:2020-7.

7. Halder S, Fett S, Locke G, et al. Impact of functional gastrointestinal disorders on health-related quality of life: a population-based case-control study. Aliment Pharmacol Ther 2004;19:233-42.

8. Canavan C, West J, Card T. The epidemiology of irritable bowel syndrome. Clin Epidemiol 2014;4:67-80.

9. Longstreth $\mathrm{G}$, Wilson A, Knight K, et al. Irritable bowel syndrome, health care use, and costs: a U.S. managed care perspective. $A m J$ Gastroenterol 2003;98:600-7.

10. Hatcher S, Arroll B. Assessment and management of medically unexplained symptoms. BMJ 2008;336:1124-8.

11. Levy R, Olden K, Naliboff B, et al. Psychosocial aspects of the functional gastrointestinal disorders. Gastroenterology 2006;130:1447-58.

12. Ryle J. An address on chronic spasmodic affections of the colon and the diseases which they simulate. Lancet 1928;212:1115-19.

13. Guthrie E, Barlow J, Fernandes L, et al. Changes in tolerance to rectal distension correlate with changes in psychological state in patients with severe irritable bowel syndrome. Psychosom Med 2004:66:578-82.

14. Dainty A. Irritable bowel syndrome: psychological comorbidities and cognitive behavioural therapy. A review of the literature. Gastrointest Nurs 2012;10:44-50.
15. Drossman D, Thompson W, Talley N, et al. Identification of subgroups of functional gastrointestinal disorders. Gastroenterol Int 1990;3:159-72.

16. Delvaux M, Denis $\mathrm{P}$, Allemand $\mathrm{H}$. Sexual and physical abuses are more frequently reported by IBS patients than by patients with organic digestive diseases or controls. Eur $J$ Gastroenterol Hepatol 1997;9:345-52.

17. Creed F, Craig T, Farmer R. Functional abdominal pain, psychiatric illness and life events. Gut 1988;29:235-42.

18. NICE. Irritable bowel syndrome in adults: diagnosis and management of irritable bowel syndrome in primary care. London: National Institute for Health and Clinical Excellence, 2008.

19. Hunt M, Moshier S, Milonova M. Brief cognitive-behavioral internet therapy for irritable bowel syndrome. Behav Res Ther 2009:47:797-802.

20. Hassett A, Gevirtz R. Nonpharmacologic treatment for fibromyalgia patient education, cognitive-behavioral therapy, relaxation techniques, and complementary and alternative medicine. Rheum Dis Clin N Am 2009;35:393-407.

21. Beck A, Rush A, Shaw B, et al. Cognitive therapy of depression: a treatment manual. New York: Guilford, 1979.

22. Craske M, Wolitzky-Taylor K, Labus J, et al. A cognitive-behavioral treatment for irritable bowel syndrome using interoceptive exposure to visceral sensations. Behav Res Ther 2011;49:413-21.

23. Drossman D, Toner B, Whitehead W, et al. Cognitive-behavioral therapy versus education and desipramine versus placebo for moderate to severe functional bowel disorders. Gastroenterology 2003:125:19-31.

24. Kennedy T, Jones R, Darnley S, et al. Cognitive behaviour therapy in addition to antispasmodic treatment for irritable bowel syndrome in primary care: randomised controlled trial. BMJ 2005; 331:435-7.

25. Zijdenbos I, De Wit N, Van der Heijden G, et al. Cochrane review: psychological treatments for the management of irritable bowel syndrome. Chichester, UK: John Wiley \& Sons, 2009.

26. Lackner J, Jaccard J, Krasner S, et al. Self-administered cognitive behavior therapy for moderate to severe irritable bowel syndrome: clinical efficacy, tolerability, feasibility. Clin Gastroenterol Hepatol 2008:6:899-906.

27. Clark D. Implementing NICE guidelines for the psychological treatment of depression and anxiety disorders: the IAPT experience. Int Rev Psychiatry 2011;23:375-84.

28. Jadad A. Randomised controlled trials: a user's guide. London: BM Books, 1998.

29. Muir-Gray J. Evidence based healthcare. New York: Churchill Livingstone, 1997.

30. Johnson B, Onwuegbuzie A. Mixed methods research: a research paradigm whose time has come. Educ Res 2004;33:14-26.

31. Creswell J, Plano-Clark V. Designing and conducting mixed methods research. What is mixed methods research? Thousand Oaks, CA: Sage, 2007

32. Scott $P$, Briggs J. A pragmatist argument for mixed methodology in medical informatics. J Mixed Methods Res 2009;3:223-41.

33. Anaf S, Sheppard L. Mixing research methods in health professional degrees: thoughts for undergraduate students and supervisors. Qual Rep 2007;12:184-92.

34. Creswell J, Plano-Clark V. Understanding mixed methods research. In: Creswell J, Plano-Clark V, eds. Designing and conducting mixed methods research. Thousand Oaks, CA: Sage, 2007: 1-19.

35. Denzin N Lincoln $Y$. The discipline and practice of qualitative research. In: Denzin N, Lincoln Y, eds. Handbook of qualitative research. Thousand Oaks, CA: Sage, 2000.

36. Pluye $\mathrm{P}$, Gagnon M, Griffiths F, et al. A scoring system for appraising mixed methods research, and concomitantly appraising qualitative, quantitative and mixed methods primary studies in mixed studies reviews. Int J Nurs Stud 2009;46:529-46.

37. Wagner K, Davidson P, Pollini R, et al. Reconciling incongruous qualitative and quantitative findings in mixed methods research exemplars from research with drug using populations. Int J Drug Policy 2012;23:54-61.

38. Spiller R, Aziz Q, Creed F, et al. Guidelines on the irritable bowel syndrome: mechanisms and practical management. Gut 2007;56:1770-98.

39. Kumano $\mathrm{H}$, Kaiya $\mathrm{H}$, Yoshiuchi $\mathrm{K}$, et al. Comorbidity of irritable bowel syndrome, panic disorder, and agoraphobia in a Japanese representative sample. Am J Gastroenterol 2004;99:370-6.

40. The Rome Foundation. To improve the lives of people with functional GI disorders. 2012. http://www.romecriteria.org/ (accessed 13 Jul 2012).

41. Ljótsson B, Hedman E, Andersson E, et al. Internet-delivered exposure-based treatment vs. stress management for irritable bowel 
syndrome: a randomized trial. Am J Gastroenterol 2011;106:1481-91.

42. Blanchard E, Lackner J, Sanders K, et al. A controlled evaluation of group cognitive therapy in the treatment of irritable bowel syndrome. Behav Res Ther 2007;45:633-48.

43. Drossman D. The functional gastrointestinal disorders and the Rome III process. Gastroenterology 2006;130:1377-90.

44. Andersson E, Ljótsson B, Smit F, et al. Cost-effectiveness of internet-based cognitive behavior therapy for irritable bowel syndrome: results from a randomized controlled trial. BMC Public Health 2011;11:215.

45. Heymann-Mönnikes I, Arnold R, Florin I, et al. The combination of medical treatment plus multicomponent behavioral therapy is superior to medical treatment alone in the therapy of irritable bowel syndrome. Am J Gastroenterol 2000;95:981-94.

46. Childress $C$. Potential risks and benefits of online psychotherapeutic interventions. 1998. http://www.ismho.org/potential_risks_and_ benefits.asp (accessed 10 Jan 2013).

47. Mohr D, Siddique J, Ho J, et al. Interest in behavioral and psychological treatments delivered face-to-face, by telephone, and by internet. Ann Behav Med 2010;40:89-98.

48. Toner B, Segal Z, Emmott S, et al. Cognitive-behavioral treatment of irritable bowel syndrome: the brain-gut connection. New York: Guilford Press, 2000.

49. IAPT. National curriculum for the education of Psychological Wellbeing Practitioners (PWPS). 2011. http://www.iapt.nhs.uk/silo/ files/national-curriculum-for-the-education-of-psychologicalwellbeing-practitioners-pwps-.pdf (accessed 28 Feb 2013).

50. Gralnek I, Hays R, Kilbourne A, et al. The impact of irritable bowel syndrome on health-related quality of life. Gastroenterology 2000;119:654-60.

51. El-Serag H, Olden K, Bjorkman D. Health-related quality of life among persons with irritable bowel syndrome: a systematic review. Aliment Pharmacol Ther 2002;16:1171-85.

52. Wiklund I, Fullerton S, Hawkey C, et al. An irritable bowel syndrome specific symptom questionnaire: development and validation. Scand J Gastroenterol 2003;38:947-54.

53. Patrick D, Drossman D, Frederick I, et al. Quality of life in persons with irritable bowel syndrome: development and validation of a new measure. Dig Dis Sci 1998;43:400-11.
54. Lydiard R, Falsetti S. Experience with anxiety and depression treatment studies: implications for designing irritable bowel syndrome clinical trials. Am J Med 1999;107:65-73.

55. Kroenke K, Spitzer R, Williams J. The PHQ-9: validity of a brief depression severity measure. J Gen Intern Med 2001; 16:606-13.

56. Löwe B, Decker O, Müller S, et al. Validation and standardization of the Generalized Anxiety Disorder screener (GAD-7) in the general population. Med Care 2008;46:266-74

57. Nespor J, Barylske J. Narrarive discourse and teacher knowledge. Am Educ J 1991;28:805-23.

58. Huberman A, Miles M. Assessing local causality in qualitative research. In: Berg D, Smith K, eds. The self in social inquiry: researching methods. Newbury Park, CA: Sage, 1988:351-81.

59. McNamara C. General guidelines for conducting interviews. 2009 http://managementhelp.org/evaluatn/intrview.htm (accessed 17 May 2012).

60. Parahoo K. Nursing research: principles, process and issues. Hampshire: Palgrave Macmillan, 2006.

61. Doyle L, Brady A, Byrne G. An overview of mixed methods research. $J$ Res Nurs 2009;14:175-85.

62. Creswell J. Mixed methods procedures. In: Creswell J, ed. Research design: qualitative, quantitative and mixed methods approaches. London: Sage, 2009:203-36.

63. Ritchie J, Spencer L. Qualitative data analysis for applied policy research. In: Bryman A, Burgess R, eds. Analysing qualitative data. London: Routledge, 1994:173-94.

64. Gunaratnam Y. Narrative and stories in health care. 2009. http:// goldsmiths.academia.edu/YasminGunaratnam/Papers/386158/ Narrative_Interviews_and_Research (accessed 17 May 2012).

65. NIHR. What is patient and public involvement in research? 2012 http://www.peopleinresearch.org/find-out-about-involvement/what-ispublic-involvement-in-research/ (accessed 6 Apr 2012).

66. NIHR. Health Technology Assessment programme. 2012. http:// www.hta.ac.uk/PPIguidance/ (accessed 6 Apr 2012).

67. Oliver $\mathrm{S}$. How can health service users contribute to the NHS research and development agenda? BMJ 1995;310:1315-18.

68. Toner B. Cognitive-behavioral treatment of functional somatic syndromes: integrating gender issues. Cogn Behav Pract 1994;1:157-78. 